\title{
Determinants Influencing Awareness and Healthy Practices among a Sample of Insulin-dependent Diabetic Egyptian Patients: A Rural Community-based Study
}

\author{
Ammal M. Metwally ${ }^{1}$ (D), Hanaa Yousof ${ }^{2}$, Monira M. Elkholy², Lobna A. Eletreby ${ }^{1}$, Abeer A. Barakat ${ }^{2}$ D, Soha M. Abd El Dayem ${ }^{3}$, \\ Mohamed Abdelrahman ${ }^{1}$ (D), Sherif M. Eldeeb ${ }^{1 *}$ iD \\ ${ }^{1}$ Department of Community Medicine Research, National Research Centre, Dokki, Cairo, Egypt; ${ }^{2}$ Department of Public Health \\ and Community Medicine, Faculty of Medicine, Cairo University, Cairo, Egypt, ${ }^{3}$ Department of Pediatric, National Research \\ Centre of Cairo, Egypt
}

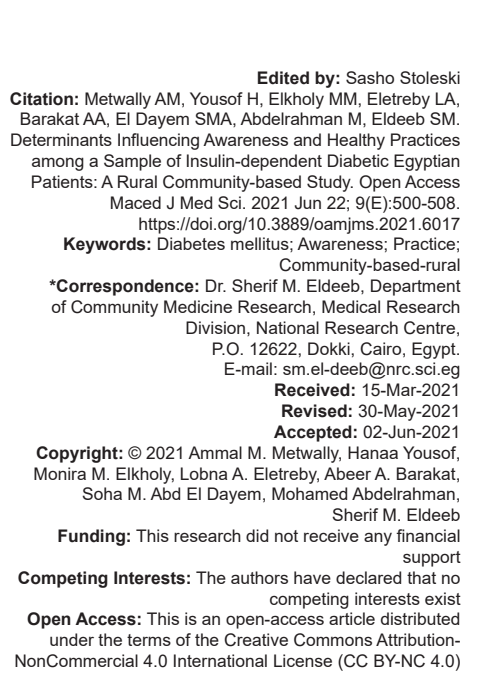

\begin{abstract}
BACKGROUND: The prevalence of diabetes mellitus (DM) is predicted to increase over the coming years.

AIM: The objectives of the study were to measure the level of awareness and healthy practices related to five healthy domains and assess the effect of different demographic characteristics, glycated hemoglobin (HbA1c) level, and body mass index (BMI) on these levels among diabetic patients in a rural Egyptian village.

METHODS: A cross-sectional study was done on 300 selected insulin-dependent diabetic patients resident in an Egyptian village. Data were collected using a questionnaire covering five domains: General disease awareness and management compliance practice, check-up, foot care, diet, and physical activity. $\mathrm{HbA} 1 \mathrm{c}$ and BMI were also measured as an impact of the management adherence.

RESULTS: The study found that more than three quarters of the participants (82.0\%) were uncontrolled or poorly uncontrolled $(\mathrm{HbA} 1 \mathrm{c}>8)$ diabetics and $76.0 \%$ were either obese or morbidly obese. Total awareness and practices percentage scores were low $(42.4 \pm 16.8 \%$ and $40.5 \pm 12.3 \%$, respectively). The linear regression model showed that high educational level had significantly positive effects on both the total awareness and practice scores as well as their domains. The study found that female participants and those having relatives with DM had significantly higher diet awareness and practice scores $(p<0.05)$. Younger age had significantly higher scores on foot care and die awareness scores, physical activity, general disease awareness, and management compliance practices domains.

CONCLUSION: The studied awareness and practice domains were inadequate. Their improvement is cornerstones to impact glycemic control of diabetics and control their health risks, especially in rural communities.
\end{abstract}

\section{Introduction}

Diabetes has been identified thousands of years ago. In fact, ancient Egyptians were the first to mention and discuss the disease, as evidenced by the Egyptian physician "Hesy-Ra" 3000 BC [1].

According to the International Diabetes Federation, it is estimated that around 40 million adults aged from 18 to 99 years are diagnosed with the disease in the Middle East and North Africa region [2], with more prevalence in the Arabian region [3]. This number is expected to increase to reach 84 million by 2045 [2].

The prevalence of diabetes in Egypt is estimated to be $15.6 \%$ with around 8.5 million suffering from diabetes in 2017. Some recent reports also indicate that there is around an extra 4.5 million undiagnosed [2], [4].
Among the rural population, the prevalence of diabetes mellitus (DM) is on the rise as evidenced by the increase in the number of cases over the past two decades [5]. Moreover, out of the $80 \%$ of the cases living in low and middle-income countries, 50\% live in rural areas [6]. In addition, studies have indicated that rural residents had low or inadequate knowledge regarding the disease [7], [8]. It was also noted that knowledge was lower among rural compared to urban population [9], [10]. This is of great impact on the outcome of this incurable disease, as previous research showed that individuals with knowledge about self-care of the disease have better control over it [11], [12]. Moreover, lifestyle modification also proved to counteract the barriers against regular physical activity, medication compliance, and following healthy nutrition [13].

In Egypt, studies have shown variation in diabetes knowledge among patients, yet the majority 
of these studies were done in health care settings and not as community ones. Previous studies indicated that diabetic patients living in rural areas had inadequate awareness and practices [14], [15], [16]. Accordingly, as an integral part of interventions targeting improving diabetic patients' health status, it is necessary to provide actual levels of knowledge and healthy practices and factors determining them.

The present study aimed to measure the level of awareness and healthy practices related to five healthy domains of diabetic patients and assess their determinants in the form of different demographic characteristics, glycated hemoglobin ( $\mathrm{HbA} 1 \mathrm{c})$ level, and body mass index (BMI) among rural diabetic patients resident in El Ibrahimia El Qeblia village, Damietta governorate, Egypt.

\section{Methods}

The study is a cross-sectional community-based one. It was approved by the ethical committee at the National Research Centre (NRC) and it was part of the project titled "Community outreach approach for having a model of a village controlled from diabetes with improved quality of life."

\section{Study duration and site}

The study was conducted over 1 year from January 2018 to January 2019. The study took place in El Ibrahimia El Qeblia, an Egyptian village situated in Damietta Governorate in North East Lower Egypt.

\section{Sample size and technique}

Using PASS v11 [17], a sample size of 283 from a population of 787 (number of diabetic patients at the village) produces a two-sided $95 \%$ confidence interval with a distance from the mean to the limits equals to 1.5 when the estimated standard deviation is 16 [18]. The sample was a simple random one.

\section{Inclusion and exclusion criteria}

Insulin-dependent patients with a history of DM aged 18 years and more for at least 1 year, as proven by interviewing the patients and by laboratory investigations, were included in the study. Patients recently diagnosed with diabetes ( $<1$ year) or with any condition interfering with proper communication or recall or evident clinical complications as well as patients on any therapy not including insulin were excluded from the study.

\section{Study tool and data collection}

The questionnaire was developed (Appendix 1 is the used study questionnaire and Appendix 2 is the English version) and subjected to pilot testing first on a small sample of diabetic patients at the NRC, Egypt. Then, pilot testing continued through questioning several diabetic patients from the study village, El Ibrahimia El Qeblia. Data from these patients were not included in the analysis. Experts' opinions were also taken from Professors of Public Health (at NRC). Both pilot tests were performed aiming at testing feasibility of the study implementation through validation (by expert opinion) of the prepared questionnaire forms, exploration of the site of the study and the system of work, and assessing the time needed to complete the interview questionnaire. Based on both pilot tests, some modifications were applied to the questionnaire.

The structured interview questionnaire collected demographic, socioeconomic, and clinical data, including age, gender, education, marital status, family history of diabetes, duration of illness, and co-morbidities.

Five domains were used for assessing diabetic patients' awareness and practices. The first was general disease awareness and management compliance practices which included knowledge of symptoms of hyper and hypoglycemia, complications, hyper and hypoglycemic coma, and the normal level of blood glucose. The healthy practices for the first domain included compliance to medication for symptoms, complication management, and physician follow-up. The second domain was for routine check-ups, including knowledge and practices for routine follow-up investigations of blood glucose, eye care, lipid profile test, and kidney and cardiac investigations. The third one was home foot care, including knowledge of the importance of foot care and self-care for foot. The fourth was knowledge and practices about the recommended number of daily meals, consumption of nutrient-rich food (vegetables, fruits, dairy products) versus energy-dense food (sweet) besides knowledge and use of harmful fats. The fifth domain was physical activity, including knowledge of the importance of physical activity, quality, and quantity for the practice of physical activity.

$\mathrm{HbA1c}$ was also assessed in percentages. Standard HbA1c for diabetics is 7\% [19]. Samples were collected in EDTA tubes. Laboratory work was done in the clinical pathology laboratory at NRC. Infection control measures were followed throughout the sampling and transport of tubes to the laboratory. $5 \mu$ of whole EDTA blood was used to assay $\mathrm{HbA} 1 \mathrm{c}$ that was measured by Labona Check A1C HbA1c Analyzer, Ceragem Medisys Inc. by boronate affinity methods.

BMI was calculated based on the equation which equals weight (in kilograms) divided by the square of the height (in meters). Weight was measured using the Seca scale. 


\section{Study outcomes}

Primary outcomes included mean awareness and mean practice scores. Secondary outcomes included mean $\mathrm{HbA} 1 \mathrm{c}$ levels and mean BMI.

\section{Data analysis}

After data cleaning, all completed questionnaires were statistically analyzed using the Statistical Package of the Social Software program (SPSS), version 20. The data were summarized using descriptive statistics where mean and standard deviation were used for quantitative variables. Number and percentage were used for qualitative values. Statistical differences between groups were tested using independent sample t-test and ANOVA (analysis of variance with Bonferroni pairwise comparison) where different letters in Rank indicate a significant difference between groups. The correlation was done between total awareness and healthy practice scores using the Pearson test. A linear regression model was done to assess the effect of different variables on the total awareness and healthy practice scores. $p<0.05$ was considered statistically significant.

\section{Scoring system}

For awareness, the maximum score was 36 ; general disease awareness: 21 , check-up: 5 , foot care: 1 , diet: 8, and physical activity: 1. Questions were given one point for right answer (No. 1, 2, 5.01, 5.02, 5.03, 5.04, 6.01, 6.02, 6.1, 7.01, 7.02, 7.03, 8.01, 8.02, 8.03, $8.04,9.01,9.02,9.03,9.04,9.05,10.01,10.02,10.03$, $10.04,10.05,11,12,13,14,15.01,15.02,15.03,15.04$, $18,21)$. As for healthy practice, the maximum score was 41; management compliance practices: 5, check-up: 5, foot care: 9, diet: 16, and physical activity: 6. Questions were either given one point for right answer (No. 2, 3, 7.1, 8.1, 9.1, 10.11, 10.12, 10.13, 10.14, 10.15, 11.2, $22.01,22.02,22.03,22.04,22.05,23)$ or a scale from 0-2 (No. 11.11, 11.12, 11.13, 11.14, 12.1, 13.1, 14.1, $16,17,18.1,19,20)$.

Total awareness and total practice percentage scores were then calculated by dividing each individual score by the maximum score for each. The same was done for each domain of awareness and practice to obtain a mean total percentage score.

As for $\mathrm{HbA1c}$, it was divided into four major categories; controlled $(\leq 7 \%)$, unsatisfactory $(>7-8 \%)$, uncontrolled $(>8-10 \%)$, and poorly uncontrolled $(>10 \%)$. BMI was also classified into four categories: Normal $\left(18.5-<25 \mathrm{~kg} / \mathrm{m}^{2}\right)$, overweight $\left(25-<30 \mathrm{~kg} / \mathrm{m}^{2}\right)$, obese $\left(30-<40 \mathrm{~kg} / \mathrm{m}^{2}\right)$, and morbid obesity $\left(\geq 40 \mathrm{~kg} / \mathrm{m}^{2}\right)$.

\section{Study duration}

The study was conducted from January 2018 to January 2019 over a total period of 1 year.

\section{Ethical aspects}

Ethical Committee of the NRC revised the study protocol (registration approval number: 16466). Written informed consent was taken from all participants. Participants' data were maintained throughout the study and the information was kept confidential. The study was conducted according to the World Medical Association Declaration of Helsinki [20].

\section{Results}

Regarding the studied 300 insulin-dependent diabetics, more than half of them were middle-aged adults $(57 \%)$ followed by old adults $(30 \%)$ and least percentage was for young adults $(13 \%)$. The mean \pm SD age of the participants was $52.7 \pm 11.2$ years with an average DM duration of $10.6 \pm 6.6$ years, giving an estimated age at diagnosis of around 40 years. Around two-thirds of the participants were females (69\%). Most of the participants were married (81\%) with around three quarters having a relative with DM as shown in Table 1. Concerning the level of education, three quarters of the cases were either illiterate or have received basic education (74.6\%). The most prevalent comorbidity was hypertension (55\%) followed by other cardiac problems and chronic liver disease. In addition, $14 \%$ of the participants were smokers. Although around two-thirds of the participants reported that they are regularly receiving DM medications $(66 \%)$, only $42 \%$ of the participants visited the physician for follow-up. The most prevalent cause for skipping a dose was financial. Table 1 also showed that upon measuring the participants' HbA1c, the mean was $10.1 \pm 2.2 \%$. Most participants had $\mathrm{HbA} 1 \mathrm{c}$ above $10 \%$. When measuring the participants' BMI, the mean was $33.3 \pm 4.8 \mathrm{~kg} / \mathrm{m}^{2}$ with two-thirds $(66 \%)$ found to be either obese or morbid obese (BMI of $30 \mathrm{~kg} / \mathrm{m}^{2}$ or more).

Table 2 shows the percentage scores for the total awareness and healthy practices and their determinants in general. The mean $\pm S D$ total awareness percentage score was $42.4 \pm 16.8$ with only $37 \%$ of the participants having scores of $50 \%$ or higher. The mean $\pm S D$ total healthy practices' percentage score was $40.5 \pm 12.3$ with only $28 \%$ of the participants having scores of $50 \%$ or more. The table also shows that although both males and females had nearly the same awareness scores, yet the practices were higher among females. Both awareness and practice scores were higher among patients having relatives with diabetes, younger age, higher educational levels, lower $\mathrm{HbA} 1 \mathrm{c}$, and normal BMI.

The mean percentages for both the total awareness and healthy practices were seen in Figure 1. The order of domains differs between the 


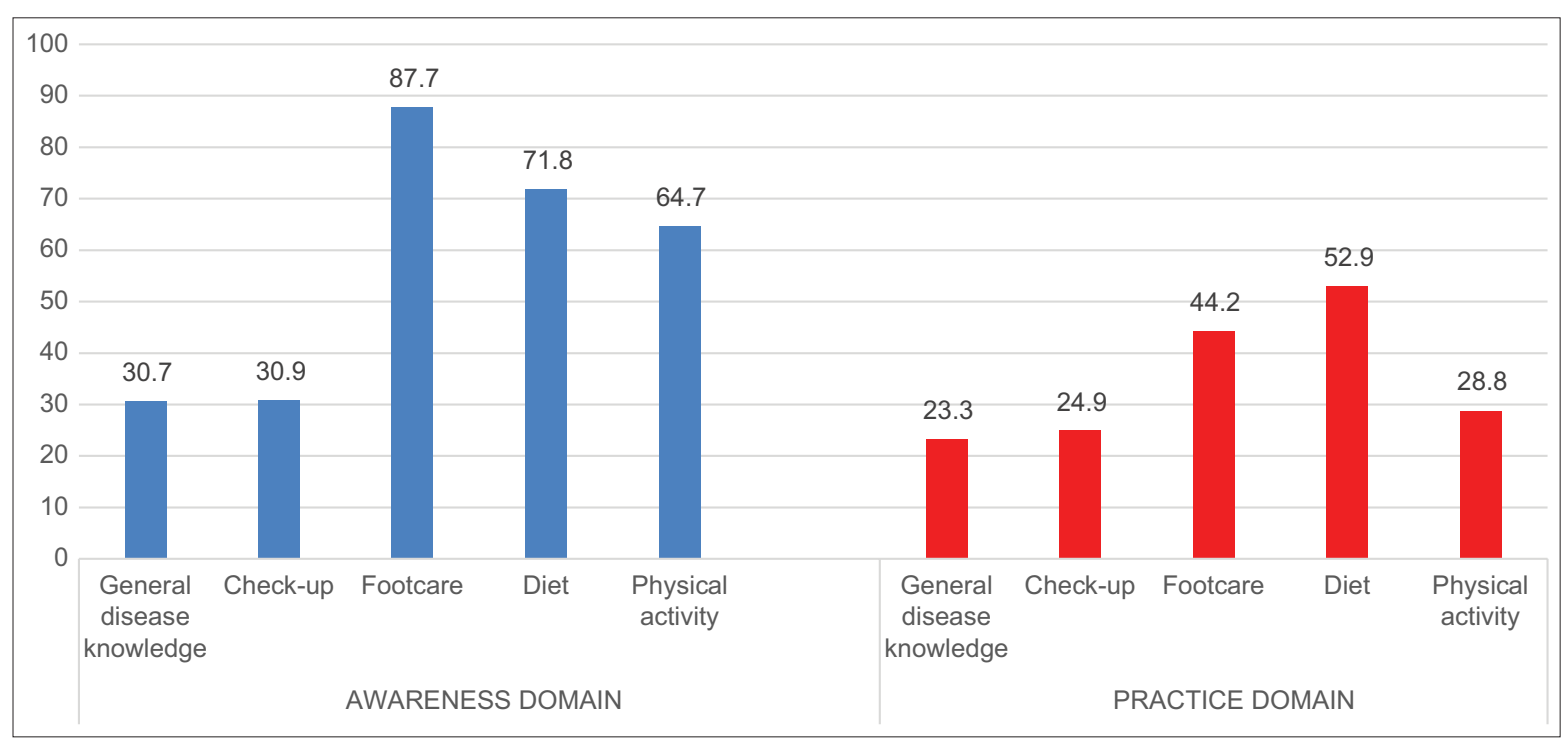

Figure 1: Mean percentage distribution of different awareness and practice domains

Table 1: Baseline sociodemographic and clinical characteristics of the studied participants

\begin{tabular}{|c|c|c|}
\hline Variables & Number (out of 300) & Percentage \\
\hline \multicolumn{3}{|l|}{ Age (years) ${ }^{\star}$} \\
\hline Young adults & 38 & 12.7 \\
\hline Middle-aged adults & 172 & 57.3 \\
\hline Old adults & 90 & 30.0 \\
\hline Mean \pm SD & $52.7 \pm 11.2$ & \\
\hline \multicolumn{3}{|l|}{ Gender } \\
\hline Male & 93 & 31.0 \\
\hline Female & 207 & 69.0 \\
\hline \multicolumn{3}{|l|}{ Marital status } \\
\hline Married & 243 & 81.0 \\
\hline Single & 57 & 19.0 \\
\hline \multicolumn{3}{|l|}{ Education } \\
\hline Illiterate & 154 & 51.3 \\
\hline Basic & 70 & 23.3 \\
\hline Secondary & 54 & 18.1 \\
\hline University & 22 & 7.3 \\
\hline \multicolumn{3}{|l|}{ Co-morbidities } \\
\hline Chronic liver diseases & 72 & 24.0 \\
\hline Chronic kidney diseases & 25 & 8.3 \\
\hline Hypertension & 166 & 55.3 \\
\hline Other heart morbidities & 75 & 25.0 \\
\hline Autoimmune diseases & 17 & 5.7 \\
\hline \multicolumn{3}{|l|}{ Having relatives with DM } \\
\hline Yes & 229 & 76.3 \\
\hline No & 71 & 23.7 \\
\hline \multicolumn{3}{|l|}{ Regular medications } \\
\hline Yes & 199 & 66.3 \\
\hline No & 101 & 33.7 \\
\hline \multicolumn{3}{|c|}{ Causes of missing dose (out of 101) } \\
\hline Financial & 62 & 61.4 \\
\hline Side effects & 23 & 22.8 \\
\hline Forgot & 11 & 10.9 \\
\hline Others & 5 & 4.9 \\
\hline \multicolumn{3}{|l|}{ Physician follow-up } \\
\hline Yes & 127 & 42.3 \\
\hline No & 173 & 57.7 \\
\hline \multicolumn{3}{|l|}{ Smoking } \\
\hline Yes & 53 & 14 \\
\hline No & 247 & 86 \\
\hline \multicolumn{3}{|l|}{ Duration of DM (years) } \\
\hline$<10$ & 143 & 47.7 \\
\hline$\geq 10$ & 157 & 52.3 \\
\hline Mean \pm SD & $10.6 \pm 6.6$ & \\
\hline \multicolumn{3}{|l|}{$\mathrm{HbA1c}\left(\%^{* \star}\right)$} \\
\hline Controlled & 22 & 7.3 \\
\hline Unsatisfactory & 32 & 10.7 \\
\hline Uncontrolled & 82 & 27.3 \\
\hline Poorly uncontrolled & 164 & 54.7 \\
\hline Mean \pm SD & $10.1 \pm 2.2$ & \\
\hline \multicolumn{3}{|l|}{ BMI $\left(\mathrm{kg} / \mathrm{m}^{2}\right)^{\star \star \star}$} \\
\hline Normal & 16 & 5.3 \\
\hline Overweight & 56 & 18.7 \\
\hline Obese & 196 & 65.3 \\
\hline Morbid obese & 32 & 10.7 \\
\hline Mean \pm SD & $33.3 \pm 4.8$ & \\
\hline
\end{tabular}

Table 2: Comparison of mean scores of awareness and practices based on sociodemographic and clinical characteristics

\begin{tabular}{|c|c|c|c|c|}
\hline \multirow[t]{2}{*}{ Studied parameters } & \multicolumn{2}{|c|}{ Total awareness score } & \multicolumn{2}{|c|}{ Total practice score } \\
\hline & Mean $\pm S D$ & $\operatorname{Rank}^{* \star \star}$ & Mean $\pm S D$ & $\operatorname{Rank}^{* \star \star}$ \\
\hline Total score & $42.4 \pm 16.8$ & & $40.5 \pm 12.3$ & \\
\hline \multicolumn{5}{|l|}{ Gender } \\
\hline Male & $42.0 \pm 18.8$ & - & $37.9 \pm 13.9^{*}$ & - \\
\hline Female & $42.6 \pm 15.9$ & & $41.6 \pm 11.3$ & \\
\hline \multicolumn{5}{|l|}{ Having relative with DM } \\
\hline Yes & $42.9 \pm 16.6$ & - & $41.3 \pm 11.9^{*}$ & - \\
\hline No & $40.6 \pm 17.6$ & & $37.7 \pm 13.1$ & \\
\hline \multicolumn{5}{|l|}{ Marital status } \\
\hline Married & $43.2 \pm 17.0$ & - & $40.8 \pm 12.4$ & - \\
\hline Single & $39.1 \pm 15.9$ & & $39.1 \pm 11.9$ & \\
\hline \multicolumn{5}{|l|}{ Smoking } \\
\hline Yes & $40.2 \pm 17.9$ & - & $37.3 \pm 14.8$ & - \\
\hline No & $42.7 \pm 16.6$ & & $41.0 \pm 11.8$ & \\
\hline \multicolumn{5}{|l|}{ Duration of DM (years) } \\
\hline$<10$ & $43.8 \pm 15.9$ & - & $41.4 \pm 13.1$ & - \\
\hline$\geq 10$ & $41.1 \pm 17.6$ & & $39.6 \pm 11.5$ & \\
\hline \multicolumn{5}{|l|}{ Age categories } \\
\hline Young adults & $49.2 \pm 14.4^{*}$ & A & $43.7 \pm 13.2^{*}$ & A \\
\hline Middle-aged & $43.3 \pm 16.3$ & A & $41.4 \pm 12.0$ & A \\
\hline Old & $37.8 \pm 17.5$ & B & $37.2 \pm 11.9$ & B \\
\hline \multicolumn{5}{|l|}{ Education } \\
\hline Illiterate & $37.8 \pm 15.6^{\star *}$ & A & $37.6 \pm 11.5^{\star \star}$ & A \\
\hline Basic & $39.8 \pm 15.0$ & A & $39.3 \pm 10.9$ & A \\
\hline Secondary & $52.0 \pm 15.5$ & B & $46.6 \pm 12.8$ & B \\
\hline University & $58.8 \pm 14.4$ & B & $49.1 \pm 11.7$ & B \\
\hline \multicolumn{5}{|l|}{$\mathrm{HbA} 1 \mathrm{c}(\%)$} \\
\hline Controlled & $55.6 \pm 16.7^{* *}$ & A & $55.2 \pm 10.6^{* *}$ & A \\
\hline Unsatisfactory & $47.2 \pm 16.6$ & $A B$ & $44.6 \pm 11.0$ & B \\
\hline Uncontrolled & $41.8 \pm 16.1$ & B & $40.2 \pm 10.1$ & $\mathrm{BC}$ \\
\hline Poorly uncontrolled & $40.0 \pm 16.4$ & B & $37.8 \pm 12.2$ & C \\
\hline \multicolumn{5}{|l|}{ BMI $\left(\mathrm{kg} / \mathrm{m}^{2}\right)$} \\
\hline Normal & $58.5 \pm 15.0^{* *}$ & A & $55.6 \pm 10.4^{* *}$ & A \\
\hline Overweight & $48.4 \pm 17.2$ & A & $45.8 \pm 12.6$ & B \\
\hline Obese & $40.2 \pm 15.1$ & B & $38.6 \pm 10.5$ & C \\
\hline Morbid obese & $37.6 \pm 19.9$ & B & $35.1 \pm 14.2$ & C \\
\hline
\end{tabular}
Diabetes mellitus, HbA1c: Glycated hemoglobin, BMl: Body mass index.

awareness and practices. Regarding awareness' domains, foot care was the highest (88\%), followed by diet $(72 \%)$, physical activity $(65 \%)$, and finally, the domains for general disease awareness and checkups $(31 \%$ each), whereas for healthy practices, diet was the highest $(53 \%)$, followed by foot care $(44 \%)$, physical activity (29\%), check-ups $(25 \%)$, and finally, management compliance practices $(23 \%)$. There was positive correlation between awareness and healthy practices $(r=0.750 ; p<0.001)$.

The effect of different determinants on the level of awareness for each domain was shown in 
Table 3. Concerning foot care domain, it was higher among younger age and individuals with $\mathrm{HbA} 1 \mathrm{c}$ of $7 \%$ or less. The diet domain was higher among females, patients having relatives with DM, younger age, and higher educational levels. The physical activity domain score was higher among married participants, patients with DM duration <10 years, younger age, and higher educational level. The mean check-ups domain was higher among patients with higher educational levels. The final domain: General disease awareness was higher among married participants, younger age, and higher educational level. Lower glycated levels and normal BMI showed higher awareness scores for all the studied domains except (physical activity and foot care, respectively).

Table 3: Comparison of mean scores of awareness based on sociodemographic and clinical characteristics in each domain

\begin{tabular}{|c|c|c|c|c|c|}
\hline Domains & $\begin{array}{l}\text { General } \\
\text { disease } \\
\text { awareness }\end{array}$ & Check-ups & Foot care & Diet & $\begin{array}{l}\text { Physical } \\
\text { activity }\end{array}$ \\
\hline Total score & $30.7 \pm 18.6$ & $30.9 \pm 28.9$ & $87.6 \pm 32.9$ & $71.8 \pm 19.2$ & $64.7 \pm 47.9$ \\
\hline \multicolumn{6}{|l|}{ Gender } \\
\hline Male & $32.6 \pm 19.5$ & $29.9 \pm 29.9$ & $83.9 \pm 37.0$ & $66.0 \pm 21.8$ & $65.6 \pm 47.8$ \\
\hline Female & $29.9 \pm 18.2$ & $31.4 \pm 28.4$ & $89.4 \pm 31.0$ & $74.4 \pm 17.3^{*}$ & $64.3 \pm 48.0$ \\
\hline \multicolumn{6}{|l|}{$\begin{array}{l}\text { Having relative } \\
\text { with DM }\end{array}$} \\
\hline Yes & $30.6 \pm 18.6$ & $31.0 \pm 29.0$ & $89.0 \pm 31.2$ & $74.0 \pm 18.4$ & $67.2 \pm 47.0$ \\
\hline No & $31.0 \pm 18.6$ & $30.7 \pm 28.7$ & $83.1 \pm 37.7$ & $64.8 \pm 21.1^{*}$ & $56.3 \pm 49.9$ \\
\hline \multicolumn{6}{|l|}{ Marital status } \\
\hline Married & $31.8 \pm 18.8$ & $30.9 \pm 28.4$ & $87.7 \pm 33.0$ & $72.0 \pm 18.9$ & $67.9 \pm 46.8$ \\
\hline Single & $26.0 \pm 16.8^{*}$ & $31.2 \pm 30.9$ & $87.7 \pm 33.1$ & $71.1 \pm 20.5$ & $50.9 \pm 50.4^{*}$ \\
\hline \multicolumn{6}{|l|}{ Smoking } \\
\hline Yes & $32.2 \pm 17.8$ & $30.5 \pm 27.3$ & $85.7 \pm 35.4$ & $64.0 \pm 23.3$ & $64.3 \pm 48.5$ \\
\hline No & $30.8 \pm 18.7$ & $30.0 \pm 29.1$ & $88.0 \pm 32.6$ & $73.1 \pm 18.1^{*}$ & $64.7 \pm 47.9$ \\
\hline \multicolumn{6}{|l|}{$\begin{array}{l}\text { Duration of DM } \\
\text { (years) }\end{array}$} \\
\hline$<10$ & $32.2 \pm 18.0$ & $32.2 \pm 26.4$ & $88.1 \pm 32.3$ & $72.5 \pm 19.4$ & $72.0 \pm 45.0$ \\
\hline$\geq 10$ & $29.3 \pm 19.1$ & $29.8 \pm 30.9$ & $87.3 \pm 33.4$ & $71.2 \pm 19.0$ & $58.0 \pm 49.5^{*}$ \\
\hline \multicolumn{6}{|l|}{ Age categories } \\
\hline Young adults & $39.2 \pm 16.1$ & $32.2 \pm 26.8$ & $97.4 \pm 16.2$ & $75.7 \pm 19.9$ & $78.9 \pm 41.3$ \\
\hline Middle-aged & $31.2 \pm 18.9$ & $31.7 \pm 28.3$ & $90.7 \pm 29.1$ & $73.1 \pm 18.4$ & $69.2 \pm 46.3$ \\
\hline Old & $26.1 \pm 17.8^{*}$ & $28.4 \pm 30.9$ & $77.8 \pm 41.8^{*}$ & $67.6 \pm 19.7^{*}$ & $50.0 \pm 50.3^{*}$ \\
\hline \multicolumn{6}{|l|}{ Education } \\
\hline Illiterate & $25.2 \pm 16.4$ & $27.7 \pm 29.2$ & $85.7 \pm 35.1$ & $69.2 \pm 19.4$ & $55.8 \pm 49.8$ \\
\hline Basic & $27.6 \pm 16.2$ & $28.3 \pm 28.2$ & $85.7 \pm 35.2$ & $70.2 \pm 20.1$ & $65.7 \pm 47.8$ \\
\hline Secondary & $42.3 \pm 17.0$ & $38.5 \pm 27.7$ & $92.6 \pm 26.4$ & $77.5 \pm 16.5$ & $77.8 \pm 42.0$ \\
\hline University & $50.9 \pm 18.3^{* *}$ & $43.6 \pm 25.9^{*}$ & $95.4 \pm 21.3$ & $80.7 \pm 15.8^{*}$ & $90.9 \pm 29.4^{*}$ \\
\hline \multicolumn{6}{|l|}{ HbA1c (\%) } \\
\hline Controlled & $42.9 \pm 21.4$ & $50.0 \pm 31.3$ & $100.0 \pm 0.0$ & $83.0 \pm 12.5$ & $86.4 \pm 35.1$ \\
\hline Unsatisfactory & $33.9 \pm 19.2$ & $38.8 \pm 28.3$ & $100.0 \pm 0.0$ & $77.7 \pm 16.4$ & $71.9 \pm 45.7$ \\
\hline Uncontrolled & $31.3 \pm 17.8$ & $29.5 \pm 29.2$ & $85.4 \pm 35.6$ & $68.8 \pm 17.3$ & $63.4 \pm 48.5$ \\
\hline $\begin{array}{l}\text { Poorly } \\
\text { uncontrolled }\end{array}$ & $28.1 \pm 17.8^{*}$ & $27.6 \pm 27.4^{*}$ & $84.8 \pm 36.1^{*}$ & $70.7 \pm 20.3^{*}$ & $61.0 \pm 48.9$ \\
\hline \multicolumn{6}{|l|}{ BMI $\left(\mathrm{kg} / \mathrm{m}^{2}\right)$} \\
\hline Normal & $47.0 \pm 18.8$ & $58.8 \pm 30.5$ & $100.0 \pm 0.0$ & $78.9 \pm 12.7$ & $93.8 \pm 25.0$ \\
\hline Overweight & $37.3 \pm 19.4$ & $35.7 \pm 29.7$ & $92.9 \pm 26.0$ & $77.0 \pm 20.9$ & $69.6 \pm 46.4$ \\
\hline Obese & $28.2 \pm 16.6$ & $29.0 \pm 27.4$ & $86.2 \pm 34.6$ & $69.8 \pm 18.5$ & $63.8 \pm 48.2$ \\
\hline Morbid obese & $26.3 \pm 22.1^{* *}$ & $20.6 \pm 26.6^{\star *}$ & $81.3 \pm 39.7$ & $71.1 \pm 20.9^{*}$ & $46.9 \pm 50.7^{*}$ \\
\hline
\end{tabular}

Table 4 shows the effect of same determinants on the healthy practices' domains. Diet domain was higher among females, patients having relatives with diabetes, non-smokers, and higher educational levels. Foot care domain was higher among patients with higher educational levels. The physical activity domain was higher among females, married patients, with disease duration of less than 10 years, and younger age. Mean percentage for the check-up domain was higher among patients with higher education levels. The management compliance practice domain was higher among married participants, younger age, and higher education. Lower glycated levels and normal BMI showed higher practices' scores for all the studied domains.
Table 4: Comparison of mean scores of practice based on sociodemographic and clinical characteristics in each domain

\begin{tabular}{|c|c|c|c|c|c|}
\hline Domains & $\begin{array}{l}\text { Management } \\
\text { compliance } \\
\text { practices }\end{array}$ & Check-ups & Foot care & Diet & $\begin{array}{l}\text { Physical } \\
\text { activity }\end{array}$ \\
\hline Total score & $23.3 \pm 18.3$ & $24.9 \pm 24.3$ & $44.2 \pm 23.0$ & $53.0 \pm 14.5$ & $28.8 \pm 18.8$ \\
\hline \multicolumn{6}{|l|}{ Gender } \\
\hline Male & $24.5 \pm 19.4$ & $23.0 \pm 25.4$ & $42.9 \pm 24.8$ & $48.6 \pm 15.2$ & $25.3 \pm 16.2$ \\
\hline Female & $22.7 \pm 17.7$ & $25.8 \pm 23.8$ & $44.8 \pm 22.3$ & $54.9 \pm 13.8^{*}$ & $30.4 \pm 19.7^{*}$ \\
\hline \multicolumn{6}{|l|}{$\begin{array}{l}\text { Having relative } \\
\text { with DM }\end{array}$} \\
\hline Yes & $23.4 \pm 18.3$ & $25.4 \pm 24.4$ & $44.8 \pm 22.1$ & $54.6 \pm 14.3$ & $29.0 \pm 18.8$ \\
\hline No & $22.8 \pm 18.3$ & $23.4 \pm 24.1$ & $42.4 \pm 26.0$ & $47.5 \pm 13.9^{\star *}$ & $28.4 \pm 19.0$ \\
\hline \multicolumn{6}{|l|}{ Marital status } \\
\hline Married & $24.2 \pm 18.7$ & $24.4 \pm 23.5$ & $44.6 \pm 22.6$ & $52.9 \pm 15.1$ & $30.2 \pm 18.3$ \\
\hline Single & $19.3 \pm 15.6^{*}$ & $27.4 \pm 27.4$ & $42.7 \pm 24.0$ & $53.1 \pm 11.6$ & $22.8 \pm 20.1^{*}$ \\
\hline \multicolumn{6}{|l|}{ Smoking } \\
\hline Yes & $24.4 \pm 17.8$ & $24.8 \pm 24.1$ & $41.8 \pm 26.7$ & $47.6 \pm 16.8$ & $26.2 \pm 17.3$ \\
\hline No & $23.4 \pm 18.4$ & $25.0 \pm 24.4$ & $44.6 \pm 22.4$ & $53.8 \pm 13.9^{*}$ & $29.3 \pm 19.0$ \\
\hline \multicolumn{6}{|l|}{$\begin{array}{l}\text { Duration of DM } \\
\text { (years) }\end{array}$} \\
\hline$<10$ & $24.5 \pm 17.7$ & $26.0 \pm 22.9$ & $44.5 \pm 23.6$ & $52.9 \pm 14.9$ & $32.8 \pm 19.3$ \\
\hline$\geq 10$ & $22.2 \pm 18.8$ & $23.9 \pm 25.6$ & $43.9 \pm 22.6$ & $52.9 \pm 14.1$ & $25.3 \pm 17.7^{*}$ \\
\hline \multicolumn{6}{|l|}{ Age categories } \\
\hline Young adults & $33.2 \pm 16.9$ & $24.7 \pm 23.0$ & $46.5 \pm 22.5$ & $54.6 \pm 18.0$ & $35.1 \pm 18.1$ \\
\hline Middle-aged & $23.6 \pm 18.3$ & $26.7 \pm 24.6$ & $45.4 \pm 21.6$ & $53.6 \pm 14.6$ & $30.2 \pm 18.3$ \\
\hline Old & $18.4 \pm 17.0^{\star \star}$ & $21.6 \pm 24.2$ & $41.0 \pm 25.7$ & $51.0 \pm 12.5$ & $23.5 \pm 19.0^{*}$ \\
\hline \multicolumn{6}{|l|}{ Education } \\
\hline Illiterate & $17.9 \pm 16.0$ & $21.4 \pm 23.9$ & $39.7 \pm 22.9$ & $51.3 \pm 14.3$ & $27.8 \pm 19.7$ \\
\hline Basic & $20.0 \pm 15.2$ & $23.7 \pm 23.2$ & $44.0 \pm 23.8$ & $51.3 \pm 13.8$ & $29.5 \pm 17.8$ \\
\hline Secondary & $34.4 \pm 16.7$ & $33.0 \pm 24.9$ & $52.7 \pm 22.0$ & $57.5 \pm 14.7$ & $29.9 \pm 17.2$ \\
\hline University & $43.6 \pm 20.1^{\text {** }}$ & $33.6 \pm 24.2^{*}$ & $56.1 \pm 12.6^{\star *}$ & $58.5 \pm 14.5^{\star}$ & $31.1 \pm 20.1$ \\
\hline \multicolumn{6}{|l|}{$\mathrm{HbA1c}(\%)$} \\
\hline Controlled & $34.5 \pm 21.5$ & $46.5 \pm 30.5$ & $64.6 \pm 13.6$ & $63.1 \pm 14.7$ & $44.7 \pm 14.9$ \\
\hline Unsatisfactory & $26.3 \pm 17.9$ & $32.5 \pm 21.4$ & $47.2 \pm 26.8$ & $56.8 \pm 11.6$ & $33.3 \pm 18.0$ \\
\hline Uncontrolled & $23.4 \pm 17.2$ & $23.9 \pm 23.6$ & $48.2 \pm 19.5$ & $51.5 \pm 13.0$ & $25.6 \pm 17.6$ \\
\hline $\begin{array}{l}\text { Poorly } \\
\text { uncontrolled }\end{array}$ & $21.1 \pm 17.9^{*}$ & $21.1 \pm 22.6^{* *}$ & $38.9 \pm 23.0^{\star *}$ & $51.5 \pm 15.1^{*}$ & $27.4 \pm 19.0^{* \star}$ \\
\hline \multicolumn{6}{|l|}{ BMI $\left(\mathrm{kg} / \mathrm{m}^{2}\right)$} \\
\hline Normal & $38.8 \pm 21.3$ & $51.3 \pm 30.1$ & $65.3 \pm 20.6$ & $60.5 \pm 13.8$ & $45.8 \pm 12.9$ \\
\hline Overweight & $29.6 \pm 18.7$ & $27.5 \pm 21.8$ & $52.2 \pm 23.1$ & $57.1 \pm 16.0$ & $34.8 \pm 14.3$ \\
\hline Obese & $21.0 \pm 16.7$ & $23.3 \pm 23.5$ & $41.7 \pm 22.0$ & $51.6 \pm 13.7$ & $26.5 \pm 18.3$ \\
\hline Morbid obese & $18.1 \pm 19.3^{\star *}$ & $17.5 \pm 22.6^{\star \star}$ & $35.4 \pm 21.4^{\star *}$ & $50.0 \pm 4.8^{\star *}$ & $24.0 \pm 18.9^{\star \star}$ \\
\hline
\end{tabular}
index.

Table 5 presents a linear regression model for predictors of the likelihood of awareness and practices which showed the variables that had a positive effect to be a high level of education ( $\beta=14.053$ and 9.382, respectively) and females for the practice $(\beta=4.115)$, whereas age (in years) had a negative effect on awareness $(\beta=-0.207)$ ( $R$ square $=0.179$ for awareness and 0.131 for practice).

Table 5: Linear regression model for the predictors of awareness and practice

\begin{tabular}{|c|c|c|c|c|c|}
\hline \multirow[t]{2}{*}{ Model } & \multirow[t]{2}{*}{$\beta$} & \multirow[t]{2}{*}{ Std. Error } & \multirow[t]{2}{*}{ Sig. } & \multicolumn{2}{|c|}{$95 \% \mathrm{Cl}$} \\
\hline & & & & Lower bound & Upper bound \\
\hline \multicolumn{6}{|l|}{ Knowledge $^{*}$} \\
\hline Constant & 49.705 & 4.603 & $<0.001$ & 40.646 & 58.764 \\
\hline Age (years) & -0.207 & 0.082 & 0.013 & -0.369 & -0.044 \\
\hline High educational level & 14.053 & 2.112 & $<0.001$ & 9.896 & 18.210 \\
\hline \multicolumn{6}{|l|}{ Practice $^{\star \star}$} \\
\hline Constant & 35.248 & 1.265 & $<0.001$ & 32.758 & 37.737 \\
\hline High educational level & 9.382 & 4.526 & $<0.001$ & 6.379 & 12.385 \\
\hline Gender (female) & 4.115 & 1.435 & 0.004 & 1.291 & 6.939 \\
\hline
\end{tabular}

\section{Discussion}

The current study is a community-based study for assessing awareness and healthy practices among 300 insulin-dependent diabetic patients and their determinants. The problem of DM was common among middle-aged adults (57.3\%), females (69.0\%), hypertensive ones $(55.3 \%)$, and those having relatives with DM (76.3\%). The mean \pm SD diabetes awareness 
and practices percentage score in the present study was $42.4 \pm 16.8 \%$ and $40.5 \pm 12.3 \%$, respectively. Although this result agrees with the results of some studies [18], [21], yet other studies reported a higher percentage [22], [23]. On the other hand, our study participants had higher scores than that reported by Balla et al. [24]. The reason behind this variation could be attributed to the fact that these studies were done in clinical or hospital setting and not community-based. In addition, such variations could be attributed to the type of counseling received, high or low cost of medications, or compliance to treatment. Moreover, the present study showed that around one-third of the participants $(33.7 \%)$ were not taking their medication on a regular basis and reported that the main reason for discontinuing treatment was the high cost of the medication $(61 \%)$ and only $42 \%$ visited physician on a regular basis for follow-up which could result in skipping dose and might be a reason for lowering practices score. Another explanation behind the low scores of awareness and practices could be attributed to the sociodemographic characteristics of the study participants. Our study being implemented in a rural village reported that most participants $(75 \%)$ were either illiterate or had only their basic education and at the same time showed that both awareness and practices tend to increase with an increase in the level of education. Furthermore, both tend also to increase among younger age which represents only $12.7 \%$ of our participants. Such findings agreed with other studies [22], [23], [25], [26]. Moreover, individuals living in rural areas tend to have lower awareness levels because these individuals might lack access to valid information, especially in Egypt [13], [16], and accordingly lower practices.

Another major factor behind the low practices score is the misconceptions and faulty information delivered to the patients where, in the present study, many participants reported that watching a TV channel commercially speaking about some sort of herbal medication is a credible source of information, believing that herbs can cure diabetes.

Furthermore, our study finding of the high level of $\mathrm{HbA1} \mathrm{c}$ with more than three-quarters of the participants $(82.0 \%)$ was uncontrolled or poorly uncontrolled $(\mathrm{HbA} 1 \mathrm{c}>8)$ reinforce the concept that the disease is poorly controlled especially taking into consideration the high prevalence of obesity among participants where over three-quarters of our participants $(76.0 \%)$ were identified as being obese (BMI $\geq 30 \mathrm{~kg} / \mathrm{m}^{2}$ ) especially that the present study was done among a rural population whose main activity is agriculture. High prevalence of obesity was also noted in another study which indicated that $65 \%$ of participants were obese [21].

Another important finding of this study showed that although the awareness score was insignificantly higher among patients with family members diagnosed with diabetes than patients without diabetic family members, yet their practices varied significantly. Balla et al., Al-maskari et al., and Niroomand et al. had supported this finding in their studies [22], [24], [27]. The South African study also agreed with the present study in the fact that having a family member with the disease was associated with higher practice score [21]. Although this is a good indicator, it is important not to totally rely on, especially in places where there are some false beliefs or misconceptions regarding the disease.

The present study was not limited to only assessing the overall awareness and practices level of diabetics but digging deeply for assessing the proved to influence the prevalence of diabetes and its control being a core risk factor for many health-related diseases. This study revealed variation within both the awareness and practices. Even though the knowledge percentage scores for foot care domain was $88 \%$ and physical activity domain was $65 \%$ which agrees with a Saudi Arabian study which reported similar results for both domains [26], yet other domains; the general disease awareness and check-ups domains showed much lower results (31\% each). Such discrepancies are important as participants seemed to have adequate diet knowledge (72\%) compared to knowledge of disease symptoms, signs, and complications as well as knowledge of the important investigations to be done. Furthermore, the check-up domains for both awareness and practices were inadequate, meaning that the participants are not aware of the importance of follow-up for the incurable disease. This also could be one of the reasons behind patients' noncompliance, especially that more than half of participants had diabetes for more than 10 years with a mean of 11 years, indicating the need for successful programs assessing reasons behind the non-compliance like other chronic diseases [13], working on interventions for increasing compliance to treatment for chronic diseases is required [28]. In addition, the relatively low awareness and practice scores among participants along with average disease duration of around 10 years raise concerns of the requirement of proper, efficient and, effective intervention models targeting diabetics, especially in rural communities. It is expected that the participants did not receive sufficient counseling as evident by the below-average awareness and practice percentage scores. These findings highlight the importance of providing community-based interventions to improve access to information which in turn reflected on both health care practices and impacted the health of diabetics. Community-based activities can lead to enhancement of the overall awareness and healthy practices among diabetics, helping proper self-control of the disease and providing support to overcome patients' problems. In Egypt, the implementation of communitybased interventions was responsive to community needs proved to positively impact different aspects of health care practices regarding infant [29], [30], [31], [32], child health and cognitive development [33], [34], [35], maternal and reproductive health [36], [37], environmental health [38], [39], and also for controlling 
diseases such as HCV [40], [41], HBV [42], [43], and end-stage renal diseases [28]. All these studies provided examples of best practices about the impact of community-based interventions on improving KAPs and health status. These findings support the future need for similar activities to achieve impact on the detected very low KAP of diabetics detected in the current study and improve their health status.

Lack of regular physical activity is another important factor where even though $44 \%$ of our study participants practiced regular mild physical activity like climbing stairs, walking, and housework, yet the percentage practicing sports or moderate to vigorous activity was extremely low $(<5 \%)$ and the overall mean physical activity score was $28.8 \%$. This could be due to the nature of the area rather than any intervention. Low level of vigorous physical activity could be ought to the high proportion of females (around two thirds) in addition to age where the majority of participants were 30 years or more and one third 60 years or more. Low level of physical activity was also reported in the Egyptian STEPS survey, where over $79 \%$ of Egyptians were not involved in vigorous physical activity [4].

The current study revealed the difference between the scores of the diet and foot care awareness domains and their corresponding practices indicating that being knowledgeable does not guarantee sound practices, but there is always a need to motivate for sound practices supporting again the need for communitybased interventions. The low level of foot care practice domain agrees with other recent studies [44].

In the present study, there was a positive correlation between level of the awareness and healthy practices $(r=0.750, p<0.001)$ which agrees with other studies $(r=0.314, p<0.001)$ [18], and $(r=0.320$, $p<0.001$ ) [22]. This also agrees with a survey done in Bangladesh among rural residents where poor or low level of knowledge was associated with poor practices and ultimately poor control and management of the disease [45], [46]. An important point to be considered is that even though there are certain predictors for the likelihood of change in both awareness and healthy practices, yet the overall effect of the model is not high. Both regression models showed that the total effect of these variables was around $18 \%$ for awareness and $13 \%$ for healthy practices, as evidenced by the $R$ square value indicating the possibility of the presence of other factors that could also affect the control of DM.
Whereas female patients, those with relatives with DM, and non-smokers had significantly higher scores concerning the awareness and practices related to the diet domain, female patients and participants with shorter duration of the disease ( $<10$ years) had significantly higher practice scores related to physical activity domain.

Marriage affected awareness and practices related to the general disease, management compliance practices, and physical activity domains.

Cost-effective targeted approaches to improve diabetic patients' awareness and healthy practices domains are required to improve self-care and impact glycemic control of diabetics, especially in rural communities.

\section{Strengths of the study}

Most of the previous studies in Egypt were done in a health care setting. The current study provided evidence drawn from the assessment of diabetics at a rural community level.

The findings of the study are considered principle and cornerstone support allowing future costeffective and targeted counseling and self-management. Moreover, this study believes that measurements and assessment of diabetics' awareness and practices of the five studied domains concerning general disease awareness and management compliance practices, check-ups, foot care, diet, and physical activity are prerequisites for achieving real change for selfmanagement and counteracting against any barrier. Accordingly, the findings of the study are considered principle and cornerstone support allowing future costeffective and targeted counseling and self-management.

\section{Limitation of the study}

This study was lacking determining the best channels of communication for applying the culturally sensitive educational tools required for diabetics' selfmanagement. These tools can be used to improve the adoption of the safe recommended behaviors by different sectors of the community to achieve the reduction of diabetes prevalence.

\section{References}

1. Khan $Y$, Hamdy O. Type 2 diabetes in the Middle East and North Africa (MENA). In: Diabetes Mellitus in Developing Countries and Underserved Communities. Cham: Springer; 2017. p. 49-61. https://doi.org/10.1007/978-3-319-41559-8_4

2. Cho N, Shaw JE, Karuranga S, Huang $Y$, da Rocha Fernandes JD, Ohlrogge AW, et al. IDF Diabetes Atlas: Global estimates of diabetes prevalence for 2017 and projections for 
2045. Diabetes Res Clin Pract 2018;138:271-81. https://doi. org/10.1016/j.diabres.2018.02.023

\section{PMid:29496507}

3. Jayawardene W, Youssefagha A, Matter S, Mokhtar A. Characteristics of Diabetes Epidemic in the Arabian Peninsula and other Muslim Countries. Ch. 1-18. Contemporary Issues in Public Health in North Africa and the Middle East; 2014.

4. WHO Egypt STEPS Survey; 2018. Available from: https://www. who.int/ncds/surveillance/steps/Egypt_STEPS_Survey_2017_ Fact_Sheet.pdf?ua=1. [Last accessed on 2020 Dec 01].

5. Hwang CK, Han PV, Zabetian A, Ali MK, Narayan KV. Rural diabetes prevalence quintuples over twenty-five years in lowand middle-income countries: A systematic review and metaanalysis. Diabetes Res Clin Pract. 2012;96(3):271-85. https:// doi.org/10.1016/j.diabres.2011.12.001

PMid:22261096

6. Guariguata L, Whiting DR, Hambleton I, Beagley J, Linnenkamp U, Shaw JE. Global estimates of diabetes prevalence for 2013 and projections for 2035. Diabetes Res Clin Pract. 2014;103(2):137-49. https://doi.org/10.1016/j. diabres.2013.11.002

PMid:24630390

7. Muninarayana C, Balachandra G, Hiremath SG, lyengar K, Anil NS. Prevalence and awareness regarding diabetes mellitus in rural Tamaka, Kolar. Int J Diabetes Dev Ctries. 2010;30(1):1821. https://doi.org/10.4103/0973-3930.60005 PMid:20431801

8. Singh A, Milton PE, Nanaiah A, Samuel P, Thomas N. Awareness and attitude toward diabetes in the rural population of Arunachal Pradesh, Northeast India. Indian J Endocrinol Metab 2012;16(Suppl 1):S83. https://doi. org/10.4103/2230-8210.94269

PMid:22701853

9. Deepa M, Bhansali A, Anjana R, Pradeepa R, Joshi SR, Joshi PP, et al. Knowledge and awareness of diabetes in urban and rural India: The Indian council of medical research India diabetes study (phase I): Indian council of medical research India diabetes 4. Indian J Endocrinol Metab. 2014;18(3):379-85. https://doi.org/10.4103/2230-8210.131191

PMid:24944935

10. Sabri AA, Qayyum MA, Saigol NU, Zafar K, Aslam F. Comparing knowledge of diabetes mellitus among rural and urban diabetics. Mcgill J Med. 2007;10(2):87-9. https://doi.org/10.26443/mjm. v10i2.741

\section{PMid: 18523544}

11. Norris SL, Engelgau MM, Narayan KM. Effectiveness of selfmanagement training in Type 2 diabetes: A systematic review of randomized controlled trials. Diabetes Care. 2001;24(3):561-87. https://doi.org/10.2337/diacare.24.3.561

PMid: 11289485

12. Duke SA, Colagiuri S, Colagiuri R. Individual patient education for people with Type 2 diabetes mellitus. Cochrane Database Syst Rev. 2009;2009(1):CD005268. https://doi. org/10.1002/14651858.cd005268.pub2

PMid:19160249

13. Metwally AM, Soliman M, Abdelmohsen AM, Kandeel WA, Saber M, Elmosalami DM, et al. Effect of counteracting lifestyle barriers through health education in Egyptian Type 2 diabetic patients. Open Access Maced J Med Sci. 2019;7(17):2886-94. https://doi.org/10.3889/oamjms.2019.624

PMid:31844454

14. El-Khawaga G, Abdel-Wahab F. Knowledge, attitudes, practice and compliance of diabetic patients in Dakahlia, Egypt. Eur J Res Med Sci. 2015;3(1):40-53.

15. Bayumi HE, Saleh HA. Knowledge, attitude and practice of diabetic patients (type II) regarding life style modification at Qena University Hospital in Upper Egypt. J Health Med Nurs. 2016;27:56-62.

16. Abdo NM, Mohamed ME. Effectiveness of health education program for Type 2 diabetes mellitus patients attending Zagazig university diabetes clinic, Egypt. J Egypt Public Health Assoc. 2010;85(3-4):113-30.

PMid:21244813

17. Hintze J. PASS 11. NCSS, LLC. Kaysville, Utah, USA. Available from: https://www.core.ac.uk/download/pdf/328824124.pdf. [Last accessed on 2019 Mar 25].

18. Fatema K, Hossain S, Natasha K, Chowdhury HA, Akter J, Khan $\mathrm{T}$, et al. Knowledge attitude and practice regarding diabetes mellitus among Nondiabetic and diabetic study participants in Bangladesh. BMC Public Health. 2017;17(1):364. https://doi.org/10.1186/s12889-017-4285-9

PMid:28446194

19. American Diabetes Association. 4. Lifestyle Management: Standards of medical care in diabetes-2018. Diabetes Care. 2018;41(Suppl 1):S38-50. https://doi.org/10.2337/dc18-s004 PMid:29222375

20. World Medical Association. World Medical Association Declaration of Helsinki. Ethical principles for medical research involving human subjects. Bull World Health Organ. 2001;79(4):373-4. https://doi.org/10.1001/jama.2013.281053 PMid: 11357217

21. Roux ML, Walsh C, Reid M, Raubenheimer J. Diabetes-related knowledge, attitude and practices (KAP) of adult patients with Type 2 diabetes mellitus in the Free State province, South Africa. South Afr J Clin Nutr. 2019;32(4):83-90. https://doi.org/ 10.1080/16070658.2018.1468536

22. Al-Maskari F, El-Sadig M, Al-Kaabi JM, Afandi B, Nagelkerke N Yeatts KB. Knowledge, attitude and practices of diabetic patients in the United Arab Emirates. PLoS One. 2013;8(1):e52857. https://doi.org/10.1371/journal.pone.0052857

PMid:23341913

23. Chen CC, Chen CL, Ko Y. The Misconceptions and determinants of diabetes knowledge in patients with diabetes in Taiwan. J Diabetes Res. 2020;2020:2953521. https://doi. org/10.1155/2020/2953521

PMid:32656263

24. Balla SA, Ahmed HA, Awadelkareem MA. Prevalence of diabetes, knowledge, and attitude of rural, population towards diabetes and hypoglycaemic event, Sudan 2013. Am J Health Res. 2014;2(6):356-60. https://doi.org/10.11648/j. ajhr.20140206.16

25. Rahaman KS, Majdzadeh R, Naieni KH, Raza O. Knowledge, attitude and practices (KAP) regarding chronic complications of diabetes among patients with Type 2 diabetes in Dhaka. Int $\mathrm{J}$ Endocrinol Metab. 2017;15(3):e12555. https://doi.org/10.5812/ ijem. 12555

PMid:29201069

26. Abouammoh NA, Alshamrani MA. Knowledge about diabetes and glycemic control among diabetic patients in Saudi Arabia. J Diabetes Res. 2020;2020:1239735. https://doi. org/10.1155/2020/1239735 PMid:32215269

27. Niroomand M, Ghasemi SN, Karimi-Sari H, KazempourArdebili S, Amiri P, Khosravi MH. Diabetes knowledge, attitude and practice (KAP) study among Iranian in-patients with Type-2 diabetes: A cross-sectional study. Diabetes Metab Syndr. 2016;10(1):S114-9. https://doi.org/10.1016/j.dsx.2015.10.006 PMid:26610404

28. Ahmed AM, Allam MF, Habil ES, Metwally AM, Ibrahiem NA, Radwan $\mathrm{M}$, et al. Development of practice guidelines for 
hemodialysis in Egypt. Indian J Nephrol. 2010;20(4):193-202. https://doi.org/10.4103/0971-4065.73450 PMid:21206681

29. Kandeel WA, Rabah TM, Zeid DA, El-Din EM, Metwally AM, Shaalan A, et al. Determinants of exclusive breastfeeding in a sample of Egyptian infants. Open Access Maced J Med Sci. 2018;6(10):1818-23. https://doi.org/10.3889/oamjms.2018.359 PMid:30455755

30. El-Din EM, Elabd MA, Nassar MS, Metwally AM, Abdellatif GA, Rabah TM, et al. The interaction of social, physical and nutritive factors in triggering early developmental language delay in a sample of Egyptian children. Open Access Maced J Med Sci. 2019;7(17):2767-74. https://doi.org/10.3889/oamjms.2019.642 PMid:31844434

31. Metwally AM, Salah El-Din EM, Shehata MA, Shaalan A, E Etreby LA, Kandeel WA, et al. Early life predictors of socioemotional development in a sample of Egyptian infants. PLoS One. 2016;11(7):e0158086. https://doi.org/10.1371/journal. pone. 0158086

PMid:27379907

32. El Din EM, Rabah TM, Metwally AM, Nassar MS, Elabd MA, Shalaan $A$, et al. Potential risk factors of developmental cognitive delay in the first two years of life. Open Access Maced J Med Sci. 2019;7(12):2024-30. https://doi.org/10.3889/oamjms.2019.566 PMid:31406549

33. Metwally AM, El-Sonbaty MM, El Etreby LA, Salah El-Din EM, Hamid NA, Hussien HA, et al. Impact of National Egyptian school feeding program on growth, development, and school achievement of school children. World J Pediatr. 2020;16(4):393400. https://doi.org/10.1007/s12519-020-00342-8

PMid:32056148

34. Metwally AM, Hanna C, Galal YS, Saleh RM, Ibrahim NA, Labib NA. Impact of nutritional health education on knowledge and practices of mothers of anemic children in El Othmanyia village-Egypt. Open Access Maced J Med Sci. 2020;8(E):45865. https://doi.org/10.3889/oamjms.2020.4570

35. Metwally AM, El-Sonbaty M, El Etreby LA, El-Din EM, Hamid NA, Hussien HA, et al. Stunting and its determinants among governmental primary school children in Egypt: A school-based cross-sectional study. Open Access Maced J Med Sci. 2020;8:650-7. https://doi.org/10.3889/oamjms.2020.4757

36. Metwally AM, Abdel-Latif GA, Mohsen A, El Etreby L, Elmosalami DM, Saleh RM, et al. Strengths of community and health facilities based interventions in improving women and adolescents' care seeking behaviors as approaches for reducing maternal mortality and improving birth outcome among low income communities of Egypt. BMC Health Serv Res. 2020;20(1):592. https://doi.org/10.1186/s12913-020-05412-1 PMid:32600377

37. Metwally AM, Saleh RM, El-Etreby LA, Salama SI, Aboulghate A, Amer HA, et al. Enhancing the value of women's reproductive rights through community based interventions in upper Egypt governorates: A randomized interventional study. Int J Equity Health. 2019;18(1):146. https://doi.org/10.1186/ s12939-019-1042-y

PMid:31533741

38. Metwally AM, Saad A, Ibrahim NA, Emam HM, El-Etreby LA Monitoring progress of the role of integration of environmental health education with water and sanitation services in changing community behaviours. Int J Environ Health Res. 2007;17(1):6174. https://doi.org/10.1080/09603120600937856 PMid:17365081

39. Metwally AM, Ibrahim NA, Saad A, Abu el-Ela MH. Improving the roles of rural women in health and environmental issues. Int J Environ Health Res. 2006;16(2):133-44. https://doi. org/10.1080/09603120500539208 PMid:16546806

40. Shiha G, Metwally AM, Soliman R, Elbasiony M Mikhail NN, Easterbrook P. An educate, test, and treat programme to reduce hepatitis $\mathrm{C}$ in Egypt: Results from a communitybased demonstration project. Lancet Gastroenterol Hepatol. 2018;3(11):778-89. https://doi.org/10.1016/ s2468-1253(18)30139-0 PMid:30030068

41. Metwally A, Mohsen A, Saleh R, Foaud W, Ibrahim N, Rabaah T, et al. Prioritizing high-risk practices and exploring new emerging ones associated with hepatitis C virus infection in Egypt. Iran J Public Health. 2014;43(10):1385094.

PMid:26060701

42. Salama II, Sami SM, Said ZN, Salama SI, Rabah TM, AbdelLatif GA, et al. Early and long term anamnestic response to HBV booster dose among fully vaccinated Egyptian children during infancy. Vaccine. 2018;36(15):2005-11. https://doi. org/10.1016/j.vaccine.2018.02.103 PMid:29530634

43. Salama II, Sami SM, Said ZN, El-Sayed MH, El Etreby LA, Rabah TM, et al. Effectiveness of hepatitis $B$ virus vaccination program in Egypt: Multicenter national project. World $\mathrm{J}$ Hepatol. 2015;7(22):2418-26. https://doi.org/10.4254/wjh.v7.i22.2418 PMid:26464758

44. Sulistyo AH, Sae-Sia W, Maneewat K. Diabetic foot care knowledge and behaviors of individuals with diabetes mellitus in Indonesia. GSTF J Nurs Health Care. 2020;5(1):1-15. https:// doi.org/10.5176/2345-7198_5.1.4

45. Islam FM, Chakrabarti R, Dirani M, Islam MT, Ormsby G, Wahab M, et al. Knowledge, attitudes and practice of diabetes in rural Bangladesh: The Bangladesh population based diabetes and eye study (BPDES). PLoS One. 2014;9(10):e110368. https://doi.org/10.1371/journal.pone.0110368 PMid:25313643

46. Horng WB, Lee CP, Chen CW. Classification of age groups based on facial features. Tamkang J Sci Eng. 2001;4(3):183-92. 